\title{
Socially Distant Medical Education in the Face of COVID-19
}

\author{
Chirag Vasavda ${ }^{1,2}$ (I) Byron K. Ho ${ }^{1,3} \cdot$ Ashwini Davison ${ }^{1,4}$
}

Accepted: 13 October 2020 / Published online: 21 October 2020

(C) International Association of Medical Science Educators 2020

\section{Introduction}

COVID-19 has profoundly altered daily life across the world. For those of us in healthcare, it has changed not only how we care for our patients and conduct research, but also how we teach and learn medicine. In an urgent effort to slow the spread of the virus, medical institutions across the world have had to suspend or dramatically limit in-person education. However, it remains essential to continue medical education across departments, residency programs, and medical schools. Not only must we continue to learn and share information to address the crisis at hand, but we must also sustain our physician pipeline. Months later, faculty are still struggling to move their curricula online, many of whom are also caring for patients combatting the novel virus. Given the possibility of future waves of infection, we must prepare to more effectively teach remotely since it is unclear when we may be back together inperson. The challenges ahead are complex and demanding, but we are optimistic that an effective transition online is possible. As we rethink and restructure medical education, we are also finding ways to better it for the future. How institutions respond to COVID-19 will not only impact this current cohort of medical students and physicians, but may also influence medical education for years to come.

Even before COVID-19, aspects of medical education had already begun to migrate online $[1,2]$. Many American medical students already watch recorded lectures at home and supplement their learning with online resources such as

Ashwini Davison

ashdavison@jhmi.edu

1 Johns Hopkins University School of Medicine, Baltimore, MD, USA

2 The Solomon H. Snyder Department of Neuroscience, Johns Hopkins University School of Medicine, Baltimore, MD, USA

3 Department of Dermatology, Johns Hopkins University School of Medicine, Baltimore, MD, USA

4 Division of General Internal Medicine, Johns Hopkins University School of Medicine, Baltimore, MD, USA
SketchyMedical [3], Pathoma [4], and UWorld [5]. Similarly, residents and attendings use online training modules and resources to prepare for licensing exams and fulfill continuing medical education (CME) requirements. However, there is more to medical education than just didactics. Many curricula integrate evidence-based strategies to enhance learning, such as team-based exercises, interactive clinical cases, and real-time quizzing [6-8]. In contrast to lectures, these other forms of pedagogy traditionally required in-person class-time. Now, COVID-19 is forcing medical education to move entirely online whether we are ready or not. This undertaking will demand significant creativity and resources, but we argue that the transition will be easier if two major needs are met: deliberate interaction between educators and learners, and an institutional infrastructure for online education. Retaining real-time personal interaction will help the changes feel less isolating, fostering effective and sustainable transitions. Institutional investments and guidance are the underlying foundations of that transition.

\section{Deliberate Interaction}

Traditional lecture and didactic content are integral components of medical education that need to be brought online. Institutions that retain lecture recordings from previous years can readily substitute those videos for in-person lectures. This is convenient but has meaningful limitations. Pre-recorded lectures risk students feeling detached, since they separate faculty from students in both time and space. Moreover, medicine changes so rapidly that content from just one year ago could already be outdated. Delivering live webinars, defined as live lectures delivered on video conferencing platforms, instead creates deliberate channels for students to interact with faculty, while still giving others flexibility to watch the recorded session later. To supplement, faculty can moderate online forums for students to pose questions related to lecture content. By building this framework, the resulting tools can also be more broadly adapted for lectures and conferences for 
residents and staff physicians. However, there are downsides to real-time lectures in a webinar format as well. One is that scheduling specific, synchronous times detracts from the flexibility that generally distinguishes online education. Additionally, synchronous meetings can further the digital divide if not all students have access to webcams, quiet spaces, and high-speed internet.

Alongside lectures, small group and team-based learning has become widely adopted by medical schools and residencies. As institutions transition their curricula online, it can be tempting to de-emphasize these small group paradigms since lectures are easier to execute remotely. However, small group teaching is not only effective but also deliberately fosters student-faculty interaction [6-8]. With the right conferencing platform [9], small group learning can also be more dynamic online than in-person. Experience and evidence suggest that team-based learning exercises can be effectively exported to an online format as well [10]. Here, students are asked to individually answer several multiple choice questions through an individual readiness assurance test (iRAT), after which they then answer the same questions in teams through a team readiness assurance test (tRAT). Alternatively, large cohorts of students can be broken up into smaller virtual rooms to work through exercises and cases before reconvening as a larger group with faculty. By shifting group dynamics to smaller groups, shyer students are empowered to interact with faculty more directly. Faculty are not actively lecturing while students are working in small groups, meaning that they are more accessible to students. Courses involving standardized patients could be reimagined with similar technologies, where students could interview patients over video conferencing software in one-on-one sessions. These sessions could be recorded for students and faculty to review afterwards.

COVID-19 also presents a long-needed opportunity to focus on and develop our telemedicine infrastructure. Technological advances have heralded greater access to healthcare for patients through telemedicine for years, but students and residents are rarely trained on how to effectively care for patients remotely $[11,12]$. The physician-patient relationship has been defined by traditional in-person communication and interpersonal interaction, much of which is not possible online. Now, institutions can develop curricula aimed at teaching students and residents on using telehealth technologies to care for, educate, and monitor patients. Students and residents can have dedicated practice at remotely interviewing patients and presenting their assessments and plans to preceptors and attendings, gaining the skills and knowledge to integrate telehealth knowledge into their practices.

Prior to COVID-19, students had already been interacting less with faculty and other peers due to a number of factors, including declining lectures attendance and a greater emphasis on preparing for board examinations. As the pandemic takes education online, these necessary professional mentor-student and student-student interactions may become even less frequent, with profound implications for students' mental health. The pandemic's social and economic consequences may likely exacerbate these issues. It is critical to deliberately carve in social and professional interactions between peers and individual faculty with students to support students' physical and mental health. Opportunities to connect can be built into coursework such as team-based assignments, jeopardy-style events, and group assessments.

Lastly, medical institutions must bring both summative and formative assessments online. Whereas summative assessments are stressful occasions because they formally evaluate students, formative assessments are low-stakes situations that often foster real-time back-and-forth between students and faculty. Additionally, summative assessments help establish whether learning objectives have been mastered, while formative assessments serve as teaching tools. Testing requires students to retrieve learned material from memory, improving long-term retention of facts and concepts. By deliberately incorporating online tools like real-time polling, faculty can create opportunities for interactive formative assessments. Formative assessments provide ongoing feedback to instructors to improve their teaching and to students to improve their learning. $[13,14]$ This will be especially helpful during the present crisis by reinforcing a new curriculum to students while allowing faculty to react to what is not working.

\section{Institutional Infrastructure}

Successfully implementing a virtual curriculum rests on institutional investment and guidance. Many physicians and educators have not used conferencing technology in this way, making the move online vulnerable to feeling chaotic and disjointed. To minimize confusion, institutional leaders should create clear protocols and guidelines for effective online teaching. This might include tutorials on navigating conferencing software, creating audience response polling, and moderating learner participation during live sessions. Standardization across an institution will facilitate a smooth transition and provide departments, educators, and speakers a common foundation for creating and delivering effective content.

A team of faculty with expertise in online education should steer the design and implementation of online medical curricula. A comprehensive team should include clinical faculty, librarians, foundational science faculty, community and population health coaches, and software programmers. Such a team would be equipped with the specialized knowledge of both medicine and online education that will be invaluable for adapting existing technologies to medical education and in developing new ones. These faculty are also best suited to advise less experienced faculty on how to export unique components of their curricula online. 
Robust information technology (IT) support teams are also essential. They can ensure that video conferencing applications run smoothly whether participants are on- or off-campus. Given the size of some departments and medical schools, IT teams should anticipate the number of connected devices for any given session and the required resources and bandwidth. IT teams can also organize educational content on online platforms by days, weeks, and blocks, easing access to online material for student and faculty.

With only a few short weeks to move entire curricula online, unexpected technical and accessibility issues will inevitably surface. These can be challenging to juggle without a centralized command center or team that can monitor and address issues as they occur. Reporting concerns to a central team can help solve problems more quickly and prevent others from experiencing the same issues. Additionally, each speaker's, school's, and department's experience can have lessons for others across the institution. New content can also be shared across different institutions, helping to address inequities in medical education by increasing accessibility to high-quality learning content. Medical education is a continuous and collaborative process, especially now.

\section{Medical Education After COVID-19}

Even during a pandemic, medical education must continue in order to ensure we are learning and sharing best practices in caring for COVID-19 patients. We must also maintain the physician pipeline. As we experiment with new pedagogical approaches to address the current crisis, some may outlast COVID-19 and become a standard part of medical education. Faculty can leverage these new educational tools to enrich traditional medical education for physicians in all career stages. With better video conferencing tools, traveling physicians will be able to participate in department grand rounds, residents and fellows at different clinical sites can have conferences together, and students can remediate missed content due to illness. Bringing more formative assessments online will also empower educators to implement more periodic testing than they have been in-person. Most importantly, we are increasing connectivity now in ways that will lower obstacles for students to build rapport with faculty in the long term. At the same time, we are also learning that some aspects a medical education do not easily translate online. In fact, some essential components of medical education are inherently hands on and may only be possible in-person, such as cadaveric dissection or learning how to perform a physical exam. We acknowledge that it has been difficult to find solutions for these aspects of medical education. Situations like these may necessitate that students meet in-person, but with students, faculty, and patients properly protected with appropriate social distancing and personal protective equipment such as masks, face shields, gloves, and gowns. Social distancing may have forced us to rethink and restructure medical education, but we are generating tools to connect, innovate, and educate that will better medicine now and in the future.

\section{Compliance with Ethical Standards}

Conflict of Interest The authors declare that they have no competing interests.

Ethical Approval N/A

Informed Consent N/A

\section{References}

1. Emanuel EJ. The inevitable reimagining of medical education. JAMA. 2020;323(12):1127-8. https://doi.org/10.1001/jama.2020. 1227.

2. Schwartzstein RM, Roberts DH. Saying goodbye to lectures in medical school - paradigm shift or passing fad? New Engl J Med. 2017;377(7):605-7. https://doi.org/10.1056/nejmp1706474.

3. SketchyMedical. https://www.sketchymedical.com. Accessed 8 April 2020.

4. Pathoma. https://www.pathoma.com. Accessed 8 April 2020.

5. UWorld. https://www.uworld.com. Accessed 8 April 2020.

6. Michaelsen LK, Watson W, Cragin JP, Fink LD. Team learning: a potential solution to the problems of large classes. J Organ Behav Educ. 1982;7(1):13-22. https://doi.org/10.1177/ 105256298200700103 .

7. Dolmans DH, Schmidt HG. What do we know about cognitive and motivational effects of small group tutorials in problem-based learning? Adv Health Sci Educ. 2006;11(4):321-36. https://doi. org/10.1007/s10459-006-9012-8.

8. Wray A, Wolff M, Boysen-Osborn M, Wiechmann W, Paradise P, Runcie E, Sudario G, Toohey S. Not another boring resident didactic conference. AEM Educ Train. 2019;4(S1). https://doi.org/10. 1002/aet2.10367

9. Marhefka S, Lockhart E, Turner D. Achieve research continuity during social distancing by rapidly implementing individual and group videoconferencing with participants: key considerations, best practices, and protocols. AIDS Behav. 2020;24:1983-9.

10. Gaber DA, Shehata MH, Amin HA. Online team-based learning sessions as interactive methodologies during the pandemic. Med Educ. 2020;54(7):666-7.

11. Perednia DA, Allen A. Telemedicine technology and clinical applications. JAMA. 1995;273(6):483-8. https://doi.org/10.1001/jama. 1995.03520300057037.

12. Bashshur RL, Reardon TG, Shannon GW. Telemedicine: a new health care delivery system. Annu Rev Public Health. 2000;21(1): 613-37. https://doi.org/10.1146/annurev.publhealth.21.1.613. Perednia.

13. Roediger HL, Butler AC. The critical role of retrieval practice in long-term retention. Trends Cogn Sci. 2011;15(1):20-7. https://doi. org/10.1016/j.tics.2010.09.003.

14. Shepard LA. Formative assessment: caveat emptor. In: Dwyer CA. The future of assessment: shaping teaching and learning. New York: Routledge; 2008.

Publisher's Note Springer Nature remains neutral with regard to jurisdictional claims in published maps and institutional affiliations. 